Revue d'histoire de l'Amérique française

\title{
Penser la mobilité, penser Montréal. La planification du tracé du réseau initial de métro, 1960-1966
}

\section{Dale Gilbert}

Volume 68, numéro 1-2, été-automne 2014

URI : https://id.erudit.org/iderudit/1032019ar

DOI : https://doi.org/10.7202/1032019ar

Aller au sommaire du numéro

Éditeur(s)

Institut d'histoire de l’Amérique française

ISSN

0035-2357 (imprimé)

1492-1383 (numérique)

Découvrir la revue

Citer cet article

Gilbert, D. (2014). Penser la mobilité, penser Montréal. La planification du tracé du réseau initial de métro, 1960-1966. Revue d'histoire de l'Amérique française, 68(1-2), 57-83. https://doi.org/10.7202/1032019ar
Résumé de l'article

Cet article examine les déterminants du choix du tracé du réseau initial de métro de Montréal. Nous démontrons que l'intégration du métro au territoire montréalais, planifiée sous la gouverne de l'administration municipale, répondit en grande partie à une volonté de faire du métro un levier de redéveloppement urbain et de protéger certaines artères des désagréments causés par sa construction. Les tensions suscitées par cette administration intransigeante envers les municipalités voisines, de même que les choix technologiques qu'elle effectua, contribuèrent également à faire en sorte que le réseau initial fut presque exclusivement limité au territoire montréalais. 


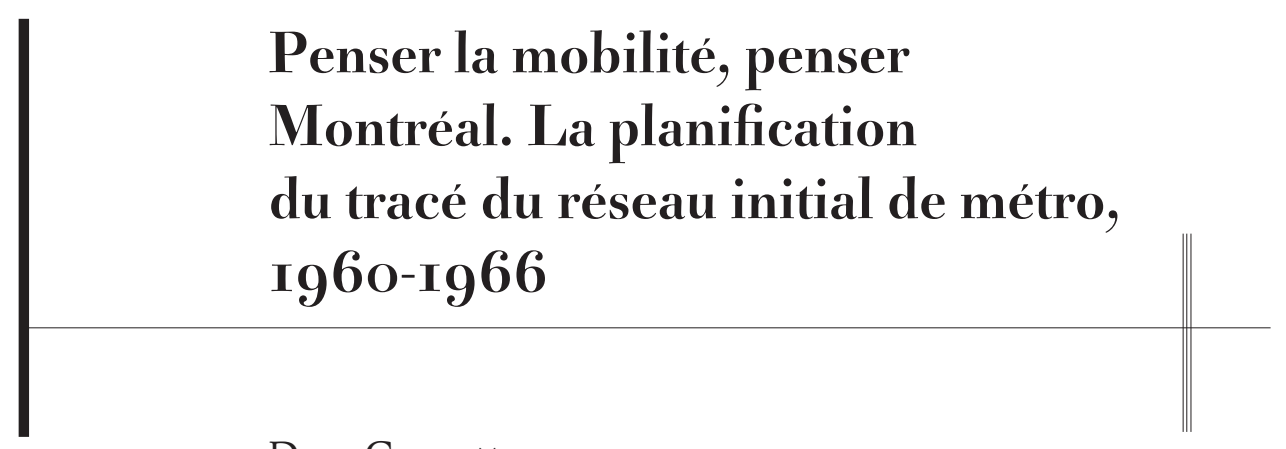

DAle Gilbert

Centre Urbanisation Culture Société

Institut national de la recherche scientifique

RÉsumé - Cet article examine les déterminants du choix du tracé du réseau initial de métro de Montréal. Nous démontrons que l'intégration du métro au territoire montréalais, planifiée sous la gouverne de l'administration municipale, répondit en grande partie à une volonté de faire du métro un levier de redéveloppement urbain et de protéger certaines artères des désagréments causés par sa construction. Les tensions suscitées par cette administration intransigeante envers les municipalités voisines, de même que les choix technologiques qu'elle effectua, contribuèrent également à faire en sorte que le réseau initial fut presque exclusivement limité au territoire montréalais.

ABSTRACT - This article addresses the factors that influenced the choice of routes for the initial subway network in Montréal. The author shows that the integration of a subway into the urban fabric was in large part guided by a twin desire of the municipal administration, in charge of the project, to use the subway as a vector of urban growth and to protect some thoroughfares from the inconveniences generated by its construction. The ensuing tensions between an intransigent administration and its neighbouring municipalities as well as the technological choices that were made explain why the subway network was almost exclusively limited to the territory of Montréal.

e 14 octobre 1966, dignitaires et invités se rassemblent à la station
Berri-De Montigny pour assister à la cérémonie d'inauguration du 
métro de Montréal ${ }^{1}$. Cette station constitue alors la plaque tournante d'un réseau souterrain de trois lignes que les Montréalais s'apprêtent à découvrir après 60 ans de débats et de projets. Le tracé de ce réseau se distingue cependant significativement de celui présenté par le maire Jean Drapeau et son bras droit Lucien Saulnier au conseil municipal en 1961, quelques mois avant le début des travaux de construction; tout comme il diffère du tracé promis lors de la campagne électorale municipale de 1960. Les rames de métro inaugurées en 1966 circulent en effet sous des artères différentes de celles qui avaient été annoncées et demeurent essentiellement, contrairement au déploiement prévu, sur le territoire de la ville de Montréal.

La région métropolitaine de Montréal franchit la barre des deux millions d'habitants au début de la décennie 1960. La croissance démographique profite à ce moment essentiellement aux banlieues, les quartiers centraux montréalais évoluant davantage sous le signe de l'exode des ménages. L'élévation d'immeubles de bureaux - notamment de gratte-ciel - au centre-ville dissimule un ralentissement de la croissance économique, qui prend fin en 1962 dans la foulée de la hausse des dépenses publiques municipales et provinciales ${ }^{2}$. Le statut de métropole canadienne, au cœur des débats sur la pertinence de construire un métro durant les années $1950^{3}$, échappe définitivement à Montréal au profit de Toronto, où roule un métro depuis 1954. La ville de Montréal est par ailleurs affligée d'importants problèmes de congestion, particulièrement au centre-ville, qui est enserré entre le fleuve Saint-Laurent et le mont Royal. Ces problèmes, présents depuis le début du siècle, s'aggravent après 1945 en raison de la multiplication rapide du nombre d'automobiles et du déclin de l'utilisation du transport collectif. Ce déclin se stabilise néanmoins au début des années $1960^{4}$. C'est dans ce contexte que Drapeau, réélu à la mairie de

1. Nous remercions Claire Poitras et les évaluateurs pour leurs judicieux commentaires, Nathalie Vachon pour la cartographie ainsi que le CRSH et le réseau Villes Régions Monde, appuyé par le FRQSC, pour leur soutien financier.

2. Mario Polèse, "Montréal économique: de 1930 à nos jours. Récit d'une transition inachevée», dans Dany Fougères, dir., Histoire de Montréal et de sa région. Tome II : De 1930 à nos jours (Québec, Les Presses de l’Université Laval, 2012), 976-982; Paul-André Linteau, Histoire de Montréal depuis la Confédération (Montréal, Boréal, 2000), 427.

3. Dale Gilbert et Claire Poitras, "Subways Are Not Outdated” Debating the Montreal Metro 19401960 ", Journal of Transport History, 36,2 (décembre 2015), à paraître.

4. Société de Transport de Montréal, Données statistiques 1952-2002 (Montréal, STM, 2003), 40. 
Montréal en $1960^{5}$, met en œuvre le projet de métro ${ }^{6}$ et lance le processus de choix de son tracé, processus au cœur de cet article.

Les travaux portant sur les métros évoquent des débats parfois houleux sur la localisation de certaines lignes et de certaines stations. Ces débats sont marqués entre autres par des tensions entre représentants des différents paliers de gouvernement, entre municipalités d'une même région métropolitaine et entre élus, professionnels et usagers ${ }^{7}$. Révélateurs de diverses visions de l'espace urbain et métropolitain et de divergences quant au rôle des métros et à l'importance du transport collectif, ils invitent à examiner en profondeur les processus d'élaboration des tracés et à porter une attention particulière à la dynamique des relations intermunicipales de même qu'aux mécanismes décisionnels et aux structures de planification mises en place.

L'étude des infrastructures de transport urbain s'est par ailleurs considérablement transformée depuis le mobility turn entrepris il y a plus d'une dizaine d'années par un groupe d'historiens français, britanniques, américains et néerlandais ${ }^{8}$. Ce virage a notamment mené les chercheurs à analyser les discours associés aux modes de déplacement, les mutations de l'espace urbain et des modes de vie induites ou espérées par la présence d'infrastructures de transport ainsi que les sentiments et les représentations découlant des déplacements ${ }^{9}$. En envisageant les infrastructures de transport comme des réalisations pétries de considérations techniques, géographiques, économiques, politiques, sociologiques, culturelles, voire

5. Jean Drapeau fut maire de Montréal de 1954 à 1957, puis de 1960 à 1986.

6. L’aménagement du réseau autoroutier montréalais se poursuit par ailleurs en parallèle de la construction du métro. Les autoroutes Bonaventure et Décarie sont notamment inaugurées six mois après son ouverture.

7. Mentionnons à ce sujet Jay Young, Searching for a Better Way: Subway Life and Metropolitan Growth in Toronto, 1942-1978, thèse de doctorat (histoire), York University, 2012; Zachary M. Schrag, The Great Society Subway. A History of the Washington Metro (Baltimore, Johns Hopkins University Press, 2006), 355 p. ; Clifton Hood, 722 Miles. The Building of the Subways and How they Transformed New York (Baltimore, Johns Hopkins University Press, 2004), 335 p.

8. Voir entre autres Mathieu Flonneau et Vincent Guigueno, «De l'histoire des transports à l'histoire de la mobilité? Mise en perspective d'un champ ", dans Mathieu Flonneau et Vincent Guigueno, dir., De l'histoire des transports à l'histoire de la mobilité? (Rennes, Presses Universitaires de Rennes, 2009), 11-24; Mathieu Flonneau, "Pour une juste place des transports dans l'histoire urbaine», Histoire urbaine, 3,11 (2004): 5-8.

9. Mentionnons Dhan Zunino Singh, «Meaningful Mobilities: The Experience of Underground Travel in the Buenos Aires Subte, 1913-1944", Journal of Transport History, 35,1 (2014): 97-113 ; Damon Scott, "When the Motorman Mayor Met the Cable Car Ladies: Engendering Transit in the City That Knows How ", Journal of Urban History, 40,1 (2014): 65-96; David Schley, "Tracks in the Streets: Railroads, Infrastructure, and Urban Space in Baltimore, 1828-1840 ", Journal of Urban History, 39,6 (2013): 10621084 ; Colin Divall et Winstan Bond, dir., Suburbanizing the Masses. Public Transport and Urban Development in Historical Perspective (Aldershot, Aldgate, 2003), 319 p. 
identitaires, les artisans de cette histoire de la mobilité placent au cœur de l'analyse le rapport entre ces infrastructures et les milieux dans lesquels elles se déploient. Une telle approche décloisonnée permet de cerner finement les forces, les aspirations et les rapports au territoire ayant façonné ces infrastructures et guidé leur intégration à leur milieu.

Young soulignait en 2012 à quel point l'historiographie du transport collectif nord-américain demeure peu développée pour la période postérieure à $1945^{10}$. Dans le domaine de la mobilité, les travaux ont en effet été surtout axés sur l'automobilisme. Le métro de Montréal, qui avec celui de Mexico (1969) est l'un des deux seuls métros inaugurés en Amérique du Nord durant les années 1960 - à une époque où à maints endroits les autorités publiques tentent de rétablir un équilibre entre automobile et transport collectif ${ }^{1}-$, ne fait pas exception à ce constat. Les études qui lui furent consacrées sont en effet en bonne partie de nature monographique et ne brossent qu'un portrait général des orientations ayant guidé l'élaboration de son tracé ${ }^{12}$.

Nous avons ainsi cherché à identifier les déterminants du choix du tracé du réseau initial de métro de Montréal. Nous avons examiné l'organisation de la gouvernance du projet, puis analysé les principes, représentations, impératifs et négociations ayant façonné le réseau. Les archives du Comité du métro, de la Commission de Transport de Montréal ${ }^{13}$ (CTM) et du Comité exécutif de la Ville de Montréal, les études et les rapports remis aux autorités ainsi que la presse francophone et anglophone $\mathrm{e}^{14}$ ont pour ce faire été mis à profit. Nous entendons démontrer dans cet article que l'intégration du métro au territoire montréalais, planifiée sous la gouverne de l'administration municipale, répondit en grande partie à une volonté de faire du métro un levier de redéveloppement urbain et de protéger certaines artères des désagréments causés par sa construction. Les tensions suscitées par cette administration intransigeante envers les municipalités voisines de même que les choix technologiques qu'elle effectua contribuèrent également à faire en sorte que le réseau initial fut presque exclusivement limité au territoire montréalais.

10. J. Young, Searching for a Better Way..., op. cit., 20-21.

11. William Middleton, Metropolitan Railways. Rapid Transit in America (Indianapolis, Indiana University Press, 2003), 107.

12. Voir notamment John Martins-Manteiga, Métro : Design in Motion/Le design en mouvement (Toronto, Dominion Modern, 2011), 443 p.; B. Clairoux, Le métro de Montréal. 35 ans déjà (Montréal, Hurtubise $\mathrm{HMH}, 2001), 160 \mathrm{p}$.

13. Organisation publique responsable du transport collectif créée en 1951.

14. Nous avons constitué un corpus de plus de 350 articles pour la période 1960-1966 grâce aux dossiers de presse thématiques, non exhaustifs, produits par le Service des Archives de la Ville de Montréal. 


\section{LA VILLE DE MONTRÉAL SEULE AUX COMMANDES DU PROJET}

Lors de la campagne électorale municipale de l'automne 1960, le Parti civique dirigé par Drapeau s'engage à supprimer la congestion au centreville grâce à la construction d'une ligne de métro est-ouest entre la rue Viau et l'avenue Atwater ${ }^{15}$, qui suivrait notamment l'axe de la rue SainteCatherine, l'artère commerciale reine de la ville. Cet axe permettrait également la desserte des principaux hôtels et gares ferroviaires ainsi que des nombreux immeubles de bureaux du secteur du boulevard Dorchester ${ }^{16}$ (aujourd'hui René-Lévesque) au sud de la rue Sainte-Catherine. La ligne disposerait par ailleurs d'un embranchement vers le quartier des affaires au centre-ville, qui emprunterait l'axe de la rue Saint-Jacques jusqu'à la Place d'Armes. Le Parti civique promet, dans une seconde étape, une ligne nord-sud suivant l'axe de la rue Saint-Denis, artère marchande d'importance située à l'est du mont Royal, entre les rues Crémazie et Sainte-Catherine ${ }^{17}$. Ce tracé s'inspire de celui recommandé par la CTM depuis 1953; il est basé sur une desserte optimale des bassins d'usagers du transport collectif ${ }^{8}$.

Conscient des bénéfices électoraux d'une telle position, Drapeau vante en public le projet de métro. Il se montre cependant beaucoup plus tiède derrière les portes closes. Lucien Saulnier, son bras droit et éventuel président du Comité exécutif, affirme dans une biographie de Drapeau parue en $1980^{19}$ que celui-ci considérait que l'ère du transport collectif était révolue au profit de l'ère de l'automobile ${ }^{20}$. Drapeau privilégie l'élargissement d'artères et la construction de voies rapides afin de soulager la congestion, un programme similaire à celui mis en pratique lors de son premier mandat ${ }^{21}$. Bien que conscient de la nécessité de développer le réseau autoroutier, Saulnier est au contraire un ardent partisan du métro.

15. Traversant ainsi les quartiers populaires Hochelaga et Sainte-Marie de même que tout le centre-ville.

16. Son élargissement en 1954-1955 en fait une artère de prestige, où sont érigés certains des immeubles phares de Montréal.

17. Traversant ainsi les quartiers Villeray et Rosemont et le Plateau Mont-Royal, habités tant par des ménages de statuts socio-économiques modestes que plus aisés, voire de la petite bourgeoisie.

18. CTM, Un système de transport en commun rapide (Montréal, CTM, 1953), 25-36.

19. Susan Purcell et Brian McKenna, Jean Drapeau (Montréal, Québec Loisirs, 1981), 143. Cet ouvrage fouillé, paru initialement en anglais en 1980, bénéficia notamment de la participation de Drapeau, qui accorda une vingtaine d'heures d'entretien.

20. Plusieurs opposants au métro soutiennent alors que ce mode de transport apparu au XIX ${ }^{\mathrm{e}}$ siècle est désuet. L'étalement urbain de même que le déclin du nombre d'usagers du transport collectif en cours à ce moment expliquent en partie cette perception.

21. La construction d'un métro faisait alors aussi partie de ses engagements électoraux. 
Ce projet doit être réalisé en priorité selon lui afin de contrer la hausse du nombre d'automobiles en circulation à Montréal.

Saulnier s'efforce rapidement de convaincre le maire après leur victoire éclatante du 24 octobre, victoire leur accordant une majorité de sièges au conseil municipal et conséquemment une grande autonomie d'action. La conversion se produit lors d'une visite du métro parisien en novembre 1960. Drapeau prend alors connaissance de la technologie de roulement sur pneumatiques, employée sur une des lignes depuis 1956. La perspective d'utiliser cette technologie française inédite en Amérique du Nord plutôt que le traditionnel système de roues d'acier sur rail transforme sa perception du métro. Celui-ci apparaît en effet pouvoir constituer désormais un symbole d'innovation pour Montréal, qui cadre bien avec la volonté de Drapeau d'ériger la ville en métropole d'envergure internationale, d'affirmer le lien historique entre le Québec et la France et de démontrer la capacité des Canadiens français à réaliser des projets distinctifs d'envergure ${ }^{22}$. Le maire quitte ainsi Paris avec la ferme intention de réaliser un métro roulant sur pneus.

Drapeau décide alors de prendre le plein contrôle du projet et notamment de l'échéancier de sa réalisation. Il souhaite en effet concrétiser rapidement cet engagement électoral, car son mandat n'est exceptionnellement que de deux ans en raison d'une réforme du mode de scrutin municipal échelonnée sur deux élections ${ }^{23}$. En décembre 1960, l’administration montréalaise se retire ainsi d'un comité conjoint formé par l'instance de gouvernance régionale, la Corporation de Montréal Métropolitain ${ }^{24}(\mathrm{CMM})$, qui étudiait différents projets de transport collectif rapide. Drapeau et Saulnier soutiennent que leur nouvelle administration ne peut participer aux décisions devant être prises considérant son absence aux réunions précédentes du comitée ${ }^{25}$. Cet habile retrait évite aux autorités montréalaises d'être liées aux conclusions de celui-ci ${ }^{26}$. Il témoigne également de la forte hostilité de Drapeau et de Saulnier, partisans de l'an-

22. Lors de l'inauguration du métro, Drapeau déclare: "Avec lui, Montréal acquiert un titre de plus parmi les grandes capitales de la terre. [...] Ville nord-américaine, sans doute, mais aussi ville française qui vient d'affirmer, une fois de plus, son caractère particulier en bâtissant un métro conçu selon le génie de la France." Archives de la Ville de Montréal (AVM), Collection Benoît Clairoux, allocution de Jean Drapeau lors de l'inauguration du métro, 14 octobre 1966. Sur le nationalisme canadien-français de Drapeau, voir notamment S. Purcell et B. McKenna, Jean Drapeau, op. cit., 151, 282.

23. P.-A. Linteau, Histoire de Montréal..., op. cit., 544.

24. Organisation (1959-1970) regroupant quinze municipalités de l'île de Montréal, incluant Montréal.

25. CMM, Rapport du sous-comité technique conjoint sur le transport en commun (Montréal, CMM, 1961), 56.

26. Le rapport du comité, déposé en janvier 1961, prône la construction de deux lignes de métro. 
nexion des municipalités de l'île à Montréal, envers la $\mathrm{CMM}^{27}$. Les deux hommes ne désirent pas que cette organisation soit en charge du projet de métro, d'autant plus que cela provoquerait des débats sur le tracé et le partage des coûts.

Les autorités montréalaises demandent officiellement au gouvernement provincial à la fin de l'année 1960 d'amender la charte de la Ville afin que leur soit accordé le pouvoir de construire un métro, qu’à ce moment ni la Ville, ni la CTM, ni la CMM ne détiennent. La Ville de Montréal s'engage à assumer seule la facture, ce qui permet d'éviter d'éventuelles longues négociations avec le gouvernement provincial ou les municipalités environnantes pouvant retarder le projet. Drapeau et Saulnier ne ferment cependant pas la porte à la possibilité que de telles contributions financières soient sollicitées après la construction. Les deux hommes réclament également de pouvoir déléguer la réalisation du projet si l'administration municipale le désire. Dans cette optique, ils demandent que le Comité exécutif de Montréal puisse nommer deux personnes au conseil de direction de la CTM. Drapeau et Saulnier s'assurent ainsi de garder le contrôle du projet s'il est confié à cette organisation, qui le réclame par ailleurs en vertu de sa mission et de l'expertise qu'elle détient.

Ces demandes sont examinées au comité des bills privés de l'Assemblée législative en janvier 1961. Si le premier ministre Jean Lesage se montre ouvert à la plupart d'entre elles, en bonne partie en raison de l'engagement de la Ville de Montréal à acquitter seule la facture ${ }^{28}$, il en va différemment pour le maire de Westmount. Soutenant que son administration est prête à payer sa part, il réclame que le métro soit construit par la CMM afin que les municipalités membres disposent d'un droit de regard sur le tracé et le financement. Plusieurs de ses confrères de la CMM approuvent au contraire la demande de la Ville de Montréal et son engagement à assumer la facture, à condition que le métro soit construit sur son territoire $^{29}$. Le 26 janvier 1961, la Ville de Montréal se voit accorder le pouvoir de construire un métro sur son territoire, de le financer seule et de délé-

27. André Lortie, «Montréal 1960. Les singularités d’un archétype métropolitain», dans André Lortie, dir., Les années 1960. Montréal voit grand (Montréal, Centre Canadien d'Architecture, 2004), 87; Stéphane Pineault, Rapports de pouvoir et enjeux métropolitains dans l'agglomération montréalaise, 1920-1961. Les problèmes de l'organisation institutionnelle, de la planification du territoire et du transport des personnes, thèse de doctorat (études urbaines), Institut national de la recherche scientifique, 2000, 319-343, 373-377; P.-A. Linteau, Histoire de Montréal..., op. cit., 550.

28. S. Pineault, Rapports de pouvoir..., op. cit., 375.

29. "II y a eu assez de comités d'études, il faut un METRO"-M. Drapeau ", La Presse, 26 janvier 1961; Pierre Laporte et Jean-Marc Laliberté, «Le métro en construction dès cette année?», Le Devoir, 27 janvier 1961; Paul Leduc, « 14.7-mile Tunnel Is Planned», Montreal Star, 27 janvier 1961. 
guer la réalisation du projet si elle le souhaite. Elle ne se voit toutefois pas investie d'un droit de regard sur le conseil de direction de la CTM. De même, les amendements adoptés ne lui permettent pas de traverser le territoire d'autres municipalités pour desservir certains quartiers montréalais. L'étude de ces questions est plutôt reportée en vue d'une réflexion plus large sur le mandat de la CMM.

Faute de pouvoir exercer un contrôle sur une CTM jouissant d'une grande autonomie, Drapeau et Saulnier décident que la Ville de Montréal ne déléguera pas son pouvoir de construction. Le Comité exécutif crée ainsi le 28 avril 1961 le Comité du métro. Présidé par le maire et formé des membres du Comité exécutif et des directeurs et de certains employés des Services municipaux d'Urbanisme, des Travaux Publics, de la Circulation, de la Voie Publique et des Finances, ce Comité est appelé, au fil de ses rencontres hebdomadaires qui s'échelonneront de mai à septembre 1961, à fixer les orientations globales du projet afin de le concrétiser rapidement $t^{30}$. Bien qu'elles se soient vu refuser la responsabilité du projet, les autorités de la CTM acceptent l'invitation à siéger au Comité, sans doute confiantes de pouvoir influencer les décisions qui y seront prises.

\section{VISIONS DIVERGENTES AU COMITÉ DU MÉTRO}

Le 5 mai 1961, trois jours après la première rencontre du Comité du métro, le Service de la Circulation dépose un rapport sur la localisation d'une éventuelle ligne de métro est-ouest. Afin de desservir les milliers d'usagers du transport collectif fréquentant les secteurs de la rue Sainte-Catherine et du boulevard Dorchester au centre-ville, le Service de la Circulation propose un tracé dans l'axe de la rue Cathcart, située entre les deux artères. Il justifie cette proposition par le fait que le coût de construction serait moins élevé que celui d'une ligne dans l'axe Sainte-Catherine et que le chantier n'entrầnerait aucune entrave à la circulation sur la grande artère commerciale de la ville ${ }^{31}$. Les membres du Comité se montrent favorables à la proposition du tracé Cathcart et l'approuvent officiellement le 17 mai $^{32}$.

Le Comité discute également au cours de ses premières réunions du système de roulement à adopter et du tracé d'une éventuelle ligne nordsud. Alors que Saulnier manifeste son intérêt pour la technologie pari-

30. AVM, Fonds du Comité exécutif (VM74), Comité du métro (S3, D70), réunion du 2 mai 1961.

31. Service de la Circulation de la Ville de Montréal, Localisation du tronçon est-ouest d’un métro pour la ville de Montréal (Montréal, Ville de Montréal, 1961), 10.

32. Les stations de bout de ligne alors approuvées dans le quartier Sainte-Marie (Frontenac) et au centre-ville (Atwater) sont situées à l'emplacement de terminus de la CTM. 
sienne de roulement sur pneus, le président-directeur général de la CTM, Guy Gauvreau, témoigne de sa préférence pour un système plus conventionnel de roues d'acier sur rail. Claude Robillard et Lucien L'Allier, respectivement directeurs des Services d'Urbanisme et des Travaux Publics, proposent, par ailleurs, d'aménager pour les fins d'un métro les voies ferrées du Canadien National (CN) courant depuis le centre-ville vers le nord de la ville via un tunnel sous le mont Royal ${ }^{33}$. Cette ligne pourrait selon eux être mise en service rapidement et serait peu coûteuse considérant l'utilisation d'infrastructures déjà existantes. Les délégués de la CTM et Saulnier se montrent cependant peu favorables à une telle ligne. Ils soutiennent qu'elle ne desservirait pas un bassin assez important d'usagers malgré le développement résidentiel important des quartiers du nord de la ville, comme Ahuntsic et Cartierville ${ }^{34}$, depuis 1945. La CTM privilégie plutôt une ligne située dans l'axe de la rue Saint-Denis qui s'étirerait du boulevard Crémazie jusqu'à la rue Saint-Jacques, effectuerait un virage sous cette artère pour traverser le quartier des affaires au centre-ville et se terminerait à l'intersection Peel/Sherbrooke, toujours au centre-ville, après un second virage. Le maire se montre, quant à lui, d'avis que les deux lignes nord-sud seront nécessaires. Des pourparlers sont ainsi entamés avec le CN. De même, la CTM et le Service d'Urbanisme entreprennent des analyses approfondies de la localisation d'une éventuelle ligne nord-sud et du système de roulement à privilégier.

Profitant de l'occasion, Saulnier demande à la CTM le 17 mai d'étudier la possibilité de changer le tracé du segment de la ligne nord-sud qui desservirait le quartier des affaires. Il suggère de remplacer l'axe SaintJacques, proposé en campagne électorale, par un axe plus au nord dans le secteur de la rue Craig (aujourd'hui Saint-Antoine). Le président du Comité exécutif souligne que cette modification aurait les mêmes avantages que le passage de Sainte-Catherine à Cathcart, soit une réduction des coûts de construction et l'absence d'entraves de longue durée à la circulation rue Saint-Jacques, principale artère du quartier des affaires. Il affirme qu'au nord de la rue Craig, il y a une «forte tendance de développement» et de "nombreux taudis à démolir ${ }^{35}$ ", révélant une volonté, d'une part, d'appuyer cette tendance par la construction d'un métro et, d'autre part, de réduire les coûts d'expropriation en ciblant des zones dévalorisées. La CTM se montre par contre peu favorable à cette modifi-

33. L'utilisation de ce tunnel par un éventuel métro est étudiée dès 1946 par le Service d’Urbanisme. 34. Après la guerre, des centaines de ménages de la classe moyenne s'installent dans ces quartiers. 35. AVM, VM74, S3, D70, réunion du 17 mai 1961. 
cation, qui rapprocherait trop selon elle les deux lignes de métro. Bien que ses délégués acceptent d'étudier la proposition de Saulnier, ils déclarent qu'un tracé dans l'axe Saint-Jacques répond mieux à leur principe de desserte optimale des bassins d'usagers du transport collectif.

Un mois plus tard, le 27 juin, les représentants du Service d'Urbanisme présentent aux membres du Comité leur évaluation du coût des expropriations pour un tracé de la ligne est-ouest passant dans l'axe Cathcart au centre-ville. Saulnier le juge trop élevé. Très soucieux du coût total du projet et faisant ainsi du montant des sommes à dépenser un critère de choix capital, il propose de réévaluer l'option Sainte-Catherine. La CTM et le Service d'Urbanisme réitèrent toutefois leur préférence pour l'axe Cathcart en soulignant que ce scénario permettrait, d'une part, d'épargner aux commerçants de la rue Sainte-Catherine les inconvénients importants qu'ils devraient subir durant la construction d'une ligne de métro et, d'autre part, d'éviter le déplacement coûteux d'un égout collecteur situé sous cette artère. Le maire Drapeau approuve en déclarant qu'il est impossible de construire le métro sous la rue Sainte-Catherine sans faire un tort considérable aux commerces. L'Allier et Guy Legault, du Service d'Urbanisme, invitent également à réfléchir à la récupération et à la revente des terrains, source éventuelle de revenus financiers. Saulnier demande ainsi au Service d'Urbanisme, comme à la CTM en mai, une étude comparative approfondie.

Au début de juillet, tant les professionnels de la CTM que ceux de la Ville de Montréal déposent des rapports sur les systèmes de roulement après avoir effectué, chacun de leur côté, une tournée européenne. Si la CTM reconnaît que le pneumatique parisien est plus silencieux que le système sur rail et qu'il est fiable et facile d'entretien, elle ne se commet pas sur le choix à faire. Les ingénieurs municipaux soulignent aussi ces avantages, mais préfèrent le système sur rail en raison des problèmes potentiels de l'utilisation hivernale du pneu en surface à Montréal et des améliorations récentes des systèmes sur rail en matière de bruit et de confort, notamment à Stockholm et à Berlin ${ }^{36}$. Saulnier rétorque que le choix à faire dépend, en ordre d'importance, du coût, des facteurs bruit et confort et, enfin, des possibilités en surface en fonction des conditions climatiques hivernales. Il invite à attendre la visite d'ingénieurs de la Régie autonome des transports parisiens (RATP) avant de choisir. Cette visite a lieu quelques semaines plus tard, au début d'août. À la suite de celle-ci et 


\section{Figure I \\ Maquette d'une voiture du métro, 1963}

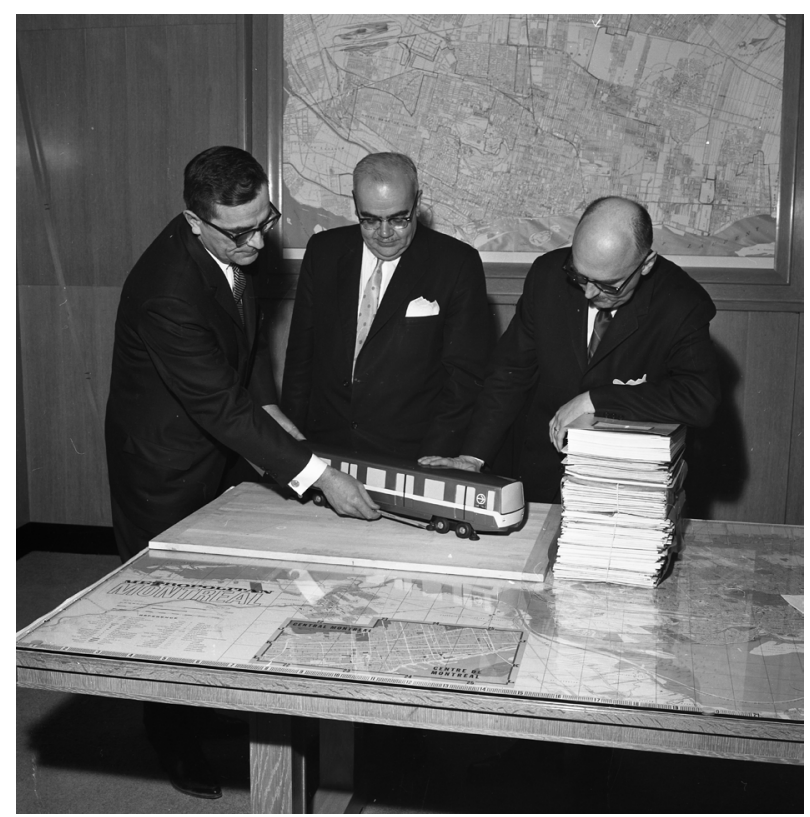

Source:AVM,VM94/M0I5-005.

malgré le scepticisme des délégués de la CTM, les membres du Comité du métro optent pour un système utilisant des voitures de petit gabarit roulant sur pneus (figure 1), jugé plus économique qu'un système conventionnel à grand gabarit sur rail ${ }^{37}$.

La CTM soumet aux membres du Comité ses recommandations sur le tracé le 19 juillet. Elle prône alors la construction d'une ligne est-ouest empruntant l'axe Cathcart au centre-ville ainsi qu'une ligne nord-sud empruntant l'axe Saint-Denis, traversant le quartier des affaires en suivant l'axe Saint-Jacques et se terminant à l'intersection Peel/Sherbrooke. Ce tracé est établi sur la base du critère d'une desserte optimale des bassins d'usagers du transport collectif. Ce critère constitue pour ses promoteurs le meilleur gage de la rentabilité du réseau. L'importance qu'on lui accorde révèle le souci d'efficacité et d'attractivité de la CTM, éventuelle exploitante du métro, dans un contexte où la fréquentation du transport

37. AVM, VM74, S3, D70, réunions des 7 et 8 août 1961. Ces choix sont également faits sur la base d'avantages techniques, par exemple en matière de courbes de virage et d'inclinaison des pentes des tunnels. 
collectif a chuté considérablement entre 1947 et $1960^{38}$. Tout en reconnaissant qu'un métro peut potentiellement influencer positivement la croissance urbaine, les professionnels du transport collectif n'accordent pas à cette influence une importance significative dans le choix du tracé, au contraire du Service d'Urbanisme, qui base ses propositions sur celle-ci, moins d'un mois plus tard.

Le Service d'Urbanisme affirme en effet dans son rapport que le choix du tracé est plus qu'une simple question de transport. Le métro a avantage notamment à être associé aux opérations de rénovation urbaine en raison de son importante influence sur la «[...] physionomie de la ville et sa vie économique ${ }^{39}$ ». Les professionnels du Service d'Urbanisme convient les autorités à construire deux lignes de métro dans une première étape, soit une ligne nord-sud s'étirant de la rue Jean-Talon à la gare ferroviaire Windsor en empruntant les axes Papineau, puis Vitré (aujourd'hui Viger) et De La Gauchetière ${ }^{40}$, et une autre ligne nord-sud ${ }^{41}$ empruntant le tunnel sous le mont Royal et reliant le nord de la ville et le centre-ville. Le rapport signale que la construction d'un métro est «[...] une occasion unique de donner à certains quartiers une vie nouvelle $e^{42}$. Le choix du corridor Vitré-De La Gauchetière, où sont localisés de nombreux taudis d'après le Service d'Urbanisme, est fait en ce sens. On croit que le métro, tout comme l'aménagement d'une autoroute, peuvent stimuler la «renaissance ${ }^{43}$ » de ce secteur.

La proposition de construire, dans une seconde étape, une ligne de métro est-ouest reliant la rue Papineau dans le quartier Sainte-Marie et le boulevard Décarie dans le quartier Notre-Dame-de-Grâce ${ }^{44}$ suit le même objectif. Au centre-ville, cette ligne emprunterait notamment l'axe de la rue Burnside, au nord de la rue Sainte-Catherine, où des expropriations sont alors en cours en vue de créer un nouveau boulevard (Maisonneuve). Cette suggestion témoigne une fois de plus de la conviction du Service d'Urbanisme que la présence d'un métro peut appuyer le redéveloppement urbain. Cette conviction, partagée également par les instances

38. On dénote une baisse de près de 114 millions de passages durant cette période. Société de Transport de Montréal, Données statistiques..., op. cit., 40; CTM, Un système de transport..., op. cit., 10.

39. Service d’Urbanisme de la Ville de Montréal, Rapport au comité du métro (Montréal, Ville de Montréal, 1961), 1-2.

40. Traversant ainsi les quartiers Rosemont et Sainte-Marie, le Plateau Mont-Royal de même qu'une bonne partie du centre-ville.

41. Ce projet fait alors l'objet de négociations entre la Ville de Montréal et le CN.

42. Service d'Urbanisme, Rapport au comité du métro..., op. cit., 2.

43. Ibid., 13.

44. Habité en bonne partie par des ménages de la classe moyenne. 


\section{Figure 2}

\section{Vue du centre-ville depuis le mont Royal, I96 I}

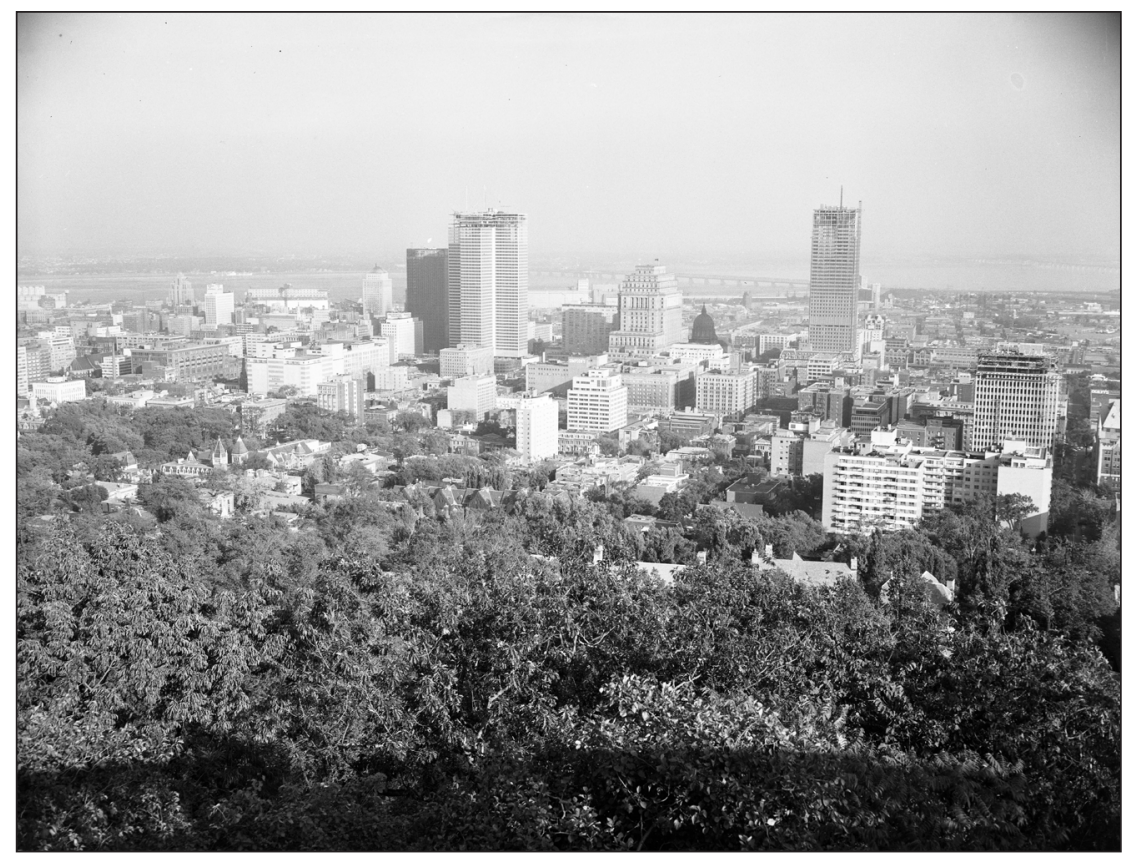

Source: BAnQ-Vieux-Montréal, Gabor Szilasi, E6/S7/SSI-P6I I 594.

planificatrices d'autres villes intéressées à la construction d'un métro au cours des années 1950 et $1960^{45}$, est sans doute alimentée par les retombées du prolongement des métros new-yorkais et londonien au cours des décennies précédentes ${ }^{46}$.

Les élus se retrouvent ainsi devant deux tracés exprimant des visions fort différentes de l'insertion du métro au territoire montréalais. Les appels de Saulnier à établir un consensus demeurent vains. Toutefois, le 22 août, une décision unanime est prise. Ce jour-là, le Comité écarte définitivement l'idée d'un tracé dans l'axe de la rue Sainte-Catherine au centre-ville, considérant qu'un chantier d'envergure de longue durée sur

45. Par exemple Santiago, au Chili, cas sur lequel travaille actuellement Andra Chastain (Yale University) dans le cadre de ses recherches doctorales. Voir son article «Moving the Masses. Toward a History of Public Transport in Postwar Latin America», dans P. Norton et al., dir., Mobility in History. The Yearbook of the International Association for the History of Transport, Traffic and Mobility. Vol. 5 (New York, Berghahn, 2014), 89-95 ; ainsi que Leopoldo Guillén, El Metropolitano de Santiago y la movilización colectiva (Santiago, Departamento de Ferrocarriles, 1952), 27 p.

46. À ce sujet, voir Peter Derrick, «Improving the Lives of the People: Rapid Transit and Residential Development in London and New York», dans Carlos López Galviz et Samuel Merrill, dir., Going Underground. New Perspectives (London, London Transport Museum, 2013), 65-71. 
cette artère entraînerait plusieurs fermetures. Cette décision témoigne d'une conscience de la situation délicate ${ }^{47}$ dans laquelle se retrouve la rue Sainte-Catherine dans un contexte marqué par le ralentissement de la croissance économique, l'étalement urbain, l'utilisation grandissante de l'automobile et l'apparition des premiers centres commerciaux en périphérie du centre-ville ${ }^{48}$. Pour la même raison, l'axe Berri sera préféré à l'axe Saint-Denis pour la ligne nord-sud. Le désir d'éviter aux artères importantes les inconvénients d'un grand chantier alimentera pour sa part le retrait de l'idée d'un axe Saint-Jacques dans le quartier des affaires.

Lors de cette même réunion du 22 août, Saulnier réagit au rapport du Service d'Urbanisme. Il affirme qu'un tracé dans l'axe Burnside pour la ligne est-ouest ne desservirait pas aussi adéquatement le centre-ville que l'axe Cathcart. Saulnier ajoute qu'un métro «[...] doit suivre le développement d'une ville et non le précéder ${ }^{49}$ ». Le maire Drapeau ainsi que les autres membres du Comité exécutif se montrent du même avis, l'un d'eux soulignant qu'en vertu de l'effervescence en matière de développement immobilier dans le secteur du boulevard Dorchester, l'axe Cathcart est préférable. La figure 2 permet à ce titre de voir deux des futurs immeubles iconiques des abords de ce boulevard, la Place Ville-Marie et la Tour CIBC, en construction à ce moment. Saulnier propose ainsi une ligne de métro est-ouest reliant les rues Frontenac et Atwater par le biais des artères Ontario, De Montigny, Cathcart et Tupper. Ce tracé est approuvé par les membres du Comité.

Néanmoins, dès la réunion suivante du Comité, Saulnier et Drapeau, très sensibles au facteur financier, se montrent ouverts au tracé Burnside, sans en être toutefois convaincus. Saulnier révèle un changement d'opinion sur le rapport entre métro et développement urbain en déclarant qu'il faut tenir compte, dans le choix du tracé, des possibilités de développement futur le long des axes choisis. Il fait également valoir que la Ville a déjà décrété des expropriations sur la rue Burnside. Drapeau soumet pour sa part aux membres du Comité l'enjeu suivant: serait-il justifié

47. Voir les réflexions de Guy Legault à ce sujet. Guy Legault, La ville qu'on a bâtie. Trente ans au service de l'urbanisme et de l'habitation à Montréal, 1956-1986 (Montréal, Liber, 2002), 54-56.

48. Mentionnons par exemple le centre commercial Rockland, inauguré en 1959. Ce contexte diffère de celui de Toronto une dizaine d'années auparavant par l'intensité des phénomènes d'étalement et d'automobilisme et par la présence de tels centres commerciaux. Au tournant des années 1950, la Toronto Transportation Commission décida de réaliser la première ligne de métro sous la rue Yonge, artère commerciale de première importance. Ce chantier ne fut pas exempt d'inconvénients pour les commerçants locaux. Les procès-verbaux du Comité du métro n’en font néanmoins pas mention. J. Young, Searching for a Better Way..., op. cit., 110-113.

49. AVM, VM74, S3, D70, réunion du 22 août 1961. 
Figure 3

Dévoilement du réseau projeté de métro, 1961

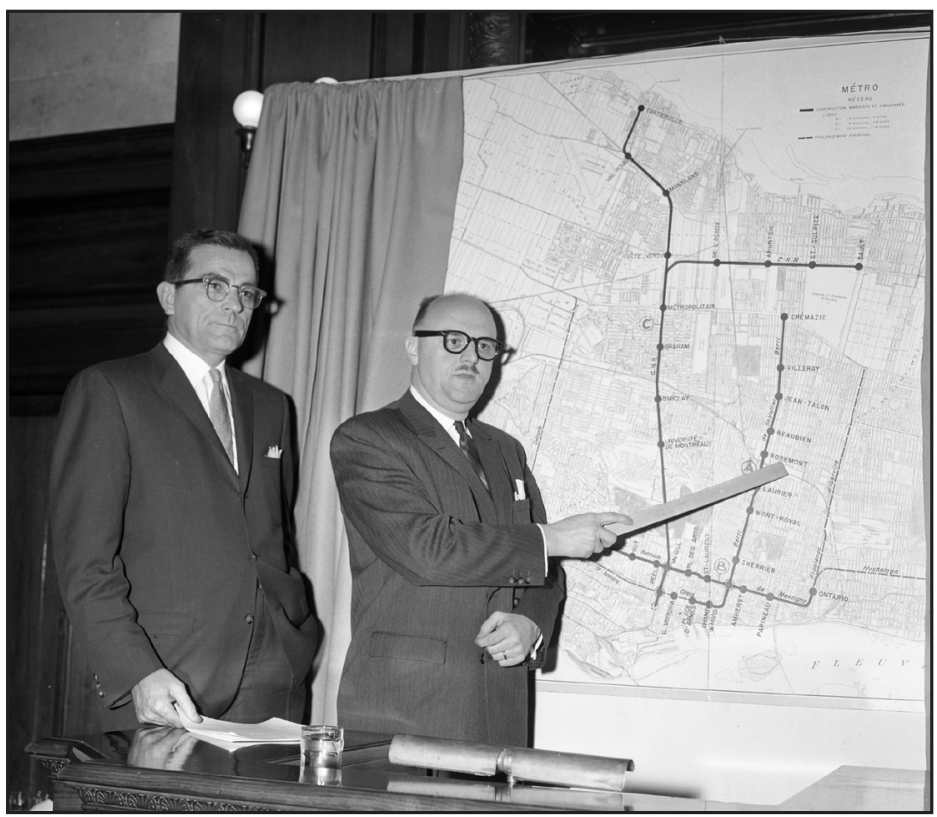

Source:AVM,VM94/ZI473-10.

d'accepter un tracé sans efficacité optimale, mais qui permettrait de réduire considérablement les coûts de construction ${ }^{50}$ ? Les délégués de la CTM, sans surprise, répondent par la négative et réaffirment que le métro doit pénétrer au cœur des zones où se trouvent les concentrations d'usagers et non pas se trouver en périphérie de celles-ci au risque de décourager les usagers.

Une dernière étude est remise au Comité lors de son ultime rencontre le 27 septembre. Le Service d'Urbanisme confirme alors que le tracé Burnside serait considérablement moins coûteux que l'option Cathcart, en raison notamment du peu d'expropriations à réaliser et de la meilleure qualité des sols à cet endroit. La CTM demeurant sur ses positions, le Comité du métro est ainsi dissous sans qu'un consensus sur le tracé ne se soit dégagé. Le maire et son bras droit demeurent toutefois fidèles à leur volonté de limiter les coûts au maximum et endossent finalement l'utili- 
sation du métro comme levier de redéveloppement urbain ${ }^{51}$, sans évacuer cependant le principe de desserte optimale prôné par la CTM.

Le 20 octobre 1961, ils dévoilent ainsi au conseil municipal et à la presse un projet de réseau de trois lignes de 150 millions de dollars, où rouleront des rames composées de voitures de petit gabarit sur pneus (figure 3). La ligne 1 (actuelle ligne verte), d'orientation est-ouest, est appelée à relier les rues Frontenac et Atwater en suivant les axes Ontario, De Montigny, Burnside et Saint-Luc. La ligne 2 (actuelle ligne orange) doit, quant à elle, relier le boulevard Crémazie à la Place d'Armes en empruntant les axes Berri, puis Craig ou Vitré, le choix final demeurant à faire.

Une ligne 3, sensiblement différente des deux autres, vient compléter le réseau. Les voitures de cette ligne doivent en effet utiliser un système de roulement sur rail traditionnel et évoluer en partie en surface. La ligne 3 est appelée à emprunter les voies ferrées du $\mathrm{CN}$ et à traverser le territoire des municipalités d'Outremont, de Mont-Royal et de Saint-Laurent ${ }^{52}$ pour ainsi relier le centre-ville aux secteurs urbanisés du nord de la ville de Montréal via le tunnel sous le mont Royal, une branche se dirigeant vers le secteur de Cartierville à l'ouest et une autre vers Ahuntsic à l'est. Saulnier déclare qu'une entente de principe a été conclue avec les autorités du $\mathrm{CN}$, mais que plusieurs détails demeurent à régler et que d'importants aménagements devront être effectués ${ }^{53}$. La réalisation et le coût de la ligne 3 sont donc dès le départ incertains, d'autant plus que la Ville de Montréal ne dispose pas du pouvoir de construire des tronçons du métro sur le territoire d'autres municipalités. Les autorités montréalaises semblent néanmoins persuadées que le gouvernement provincial leur accordera rapidement ce pouvoir. Saulnier affirme en effet au moment du dévoilement qu'il est possible que la ligne 3 soit mise en service avant les autres.

\section{D'UNE LIGNE SOUS LA MONTAGNE À UNE LIGNE SOUS LE FLEUVE}

La réaction de la population, de la presse, de la Chambre de commerce de Montréal et de différentes associations de gens d'affaires ${ }^{54}$ au tracé

51. Ce principe ainsi que le climat montréalais conduiront également les autorités à décider que les bouches d'accès ne seraient pas situées sur les trottoirs, mais bien en bordure de ceux-ci et intégrées au tissu bâti. Cette décision pave la voie à un programme de location des droits aériens au-dessus des stations, qui ne connaîtra que peu de succès à ses débuts et dont l'examen fouillé demeure à faire.

52. Banlieues faisant partie de la CMM et comptant une forte proportion de ménages aisés.

53. L'incertitude quant au tracé de la ligne 3 au centre-ville explique selon Clairoux que les secteurs du square Victoria et des gares ferroviaires Centrale et Windsor ne soient pas couverts par celle-ci ou la ligne 2 dans le réseau projeté d’octobre 1961. B. Clairoux, Le métro de Montréal..., op. cit., 31.

54. Comme celle de la municipalité de Saint-Laurent par exemple. "M. Jacques Viau: "C'est 'la' solution idéale" ", La Presse, 24 octobre 1961. 
dévoilé ainsi qu'aux principes ayant guidé son choix est globalement favorable. Soutenant qu'il y a d'autres bassins substantiels de population à desservir, plusieurs citoyens, éditorialistes et conseillers municipaux indépendants en faveur du projet de métro considèrent toutefois que le réseau proposé est trop limité et que les Montréalais accepteraient que la facture soit plus élevée afin que le réseau soit davantage étendu. Lors des séances spéciales du conseil municipal visant à étudier le projet, les conseillers Edmund Asselin et Frank Donnelly réclament, par exemple, que la ligne 1 rejoigne le quartier Notre-Dame-de-Grâce à l'ouest et le boulevard Pie-IX dans le quartier Hochelaga à l'est. L'administration oppose cependant une fin de non-recevoir à ces demandes en invoquant l'importance de respecter un équilibre entre les coûts de construction et d'opération et les revenus d'exploitation. Le 3 novembre 1961, les crédits nécessaires (132 millions de dollars $\left.{ }^{55}\right)$ à la réalisation des lignes 1 et 2 sont votés par une large majorité. Le vote des crédits associés à la ligne 3 est quant à lui reporté en raison de l'incertitude entourant plusieurs aspects de la réalisation de celle-ci.

Lors de l'annonce du 20 octobre, Saulnier déclare que les municipalités de banlieue seront appelées à participer financièrement à la construction du métro. Conscients qu'une demande de contribution directe se moyennera par un droit de regard sur le projet, ce que proposera en effet le maire d'Outremont Romuald Bourque ${ }^{56}$, Drapeau et Saulnier demandent au gouvernement provincial en mars 1962 que le montant de la taxe de vente perçue à Montréal ne soit plus partagé entre les quinze municipalités de la CMM. Les deux hommes souhaitent que ce montant reste entre les mains de l'administration montréalaise. Ils réclament également que la charte de la Ville de Montréal soit amendée afin qu’y soit inscrit le pouvoir de construire, de posséder et d'exploiter un métro sur le territoire des municipalités voisines. En outre, l'administration montréalaise désire obtenir le pouvoir d'aménager dans ces municipalités des parcs de stationnement aux endroits de son choix et des espaces commerciaux dans les stations ou au-dessus de celles-ci. On demande aussi que toutes les infrastructures associées au métro, de même que les espaces commerciaux, soient exemptés de taxes. Ces requêtes pour le moins exigeantes sont formulées au moment où Drapeau et Saulnier, aux visées annexionnistes

55. Incluant notamment l'achat du matériel roulant.

56. Bourque rend la participation financière de sa municipalité conditionnelle à ce que le métro soit construit sous l'autorité de la CMM, ce qui assurerait aux municipalités membres un pouvoir décisionnel. "Outremont toujours prêt à participer», La Presse, 21 octobre 1961. 
avouées, tentent d'obtenir le contrôle de la CMM par le biais d'une réforme de la représentation des municipalités au sein de l'organisation.

Les représentants des municipalités d'Outremont, de Mont-Royal et de Saint-Laurent se montrent ouverts à l'idée que le métro traverse leur territoire lors de l'étude, par le comité des bills privés, des amendements demandés. Ils manifestent cependant le désir de pouvoir approuver les plans et s'opposent vigoureusement à la possibilité que la Ville de Montréal puisse construire sans leur autorisation des stationnements et des édifices commerciaux sur leur territoire. Ils réclament également de pouvoir récolter des revenus de taxes sur ces infrastructures et ces édifices. Après quelques jours de négociations, une entente est conclue et entérinée par le gouvernement provincial.

Le 21 mars 1962, la Ville de Montréal se voit ainsi accorder le pouvoir de construire, de posséder et d'exploiter un métro le long des voies du CN dans les municipalités d'Outremont, de Mont-Royal et de SaintLaurent, le tout étant sujet à la conclusion d'une entente avec le $\mathrm{CN}$ pour l'aménagement des voies. Les amendements adoptés stipulent également que l'aménagement de parcs de stationnement est conditionnel à l'accord des autorités locales et que la Ville de Montréal ne peut aménager des locaux commerciaux qu'à l'intérieur des stations. Ces locaux sont soumis à la taxation municipale locale. Les infrastructures propres au métro, dont les stations, en sont toutefois exemptées ${ }^{57}$. Finalement, le gouvernement refuse que la Ville de Montréal garde pour elle seule les revenus de la taxe de vente prélevée sur son territoire.

Un an plus tard, en mars 1963, Drapeau et Saulnier reviennent à la charge en demandant l'autorisation de vendre ou de louer les droits aériens au-dessus des stations du réseau afin qu'y soient érigés des édifices de fonctions diverses. Ils réclament aussi que sur le territoire des municipalités voisines, le produit des taxes sur ces édifices soit consacré, du moins en majeure partie, aux coûts d'entretien du métro. L'opposition d'Outremont et de Mont-Royal conduit cependant au rejet de la partie des requêtes touchant les municipalités de banlieue ${ }^{58}$. Les autorités montréalaises se retrouvent ainsi avec le pouvoir de construire la ligne 3, mais ne disposent d'aucun levier pour financer sa réalisation dans les municipalités

57. Jean-Marc Laliberté, «Montréal reste maître de son projet de métro », Le Devoir, 22 mars 1962.

58. Jacques Francoeur, «En voulant jouer aux "finfins", Outremont, Westmount et Mt-Royal perdraient le métro!», Dimanche-Matin, 17 mars 1963. 
voisines, qui rendent l'obtention d'un droit de regard sur le projet conditionnelle à toute contribution ${ }^{59}$.

Les pourparlers avec le CN, par ailleurs, se poursuivent entre 1961 et 1963 en vue de la réalisation de la ligne 3. La construction de voies additionnelles apparaît rapidement nécessaire afin de faciliter la cohabitation du métro et des trains réguliers. En outre, la CTM convainc les autorités montréalaises peu après l'annonce du 20 octobre 1961 d'étudier de concert avec le CN la possibilité d'aménager les voies de chemin de fer afin d'y faire rouler des voitures sur pneus. Elle soutient que l'utilisation de deux systèmes de roulement entraînerait des coûts d'entretien supplémentaires et gênerait la répartition adéquate des rames de métro sur le réseau au gré des besoins. L'adaptation des voies s'avère cependant particulièrement complexe. Un comité, spécialement créé par la CTM pour étudier cette question, souligne en mai 1962 les coûts très élevés des travaux requis et de l'entretien hivernal des voies en surface. Les conclusions du comité, comme celles d'autres rapports réalisés sur cette question, sont défavorables à l'aménagement des voies ferrées pour y faire rouler des voitures sur pneus $^{60}$. Ainsi, en raison de l'impasse dans laquelle se retrouve l'administration montréalaise au sujet du système de roulement à adopter pour les rames de la ligne 3 et de l'incapacité de cette même administration de conclure une entente de nature financière avec les municipalités traversées, qui lui permettrait de ne pas assumer seule une facture s'annonçant plus élevée que prévu, cette ligne 3 apparaît en difficulté au printemps 1963, au moment où est soumise l'idée d'une autre ligne de métro.

En novembre 1962, un mois après la victoire éclatante de Drapeau et de son parti aux élections municipales et six mois après le début des travaux de construction du métro ${ }^{61}$, la Ville de Montréal obtient l'organisation de l'Exposition universelle de 1967. Le choix du site des îles du SaintLaurent est arrêté à la fin de mars 1963 et quelques jours plus tard, l'administration montréalaise révèle à la population son projet de construire une ligne de métro reliant Montréal à la rive sud du fleuve et

59. Les autorités de Montréal et de Westmount concluent néanmoins une entente pour l'aménagement de la station Atwater, dont les installations vont se déployer de chaque côté de leur frontière commune. Cette entente prévoit le versement par Westmount d'une contribution aux dépenses d'opération du métro. ASTM, S6, Série Imprimés et journaux (10), Imprimés de la RATP/Bulletin d’information et de documentation (2,1), brochure Le métropolitain de Montréal, mars-avril 1967.

60. ASTM, S6, Série 7, Planification et coordination/Études du réseau/Comité d'intégration (2.2,5), rapport du Integration Task Force, 23 mai 1962.

61. Ces travaux, lancés le 23 mai 1962, furent supervisés par le Bureau du métro, organisation municipale créée à l'été 1961 et placée sous la direction de L’Allier. Ce dernier sera également président-directeur général de la CTM de 1964 à 1973. 
Figure 4

Réseau modifié de métro, 1963

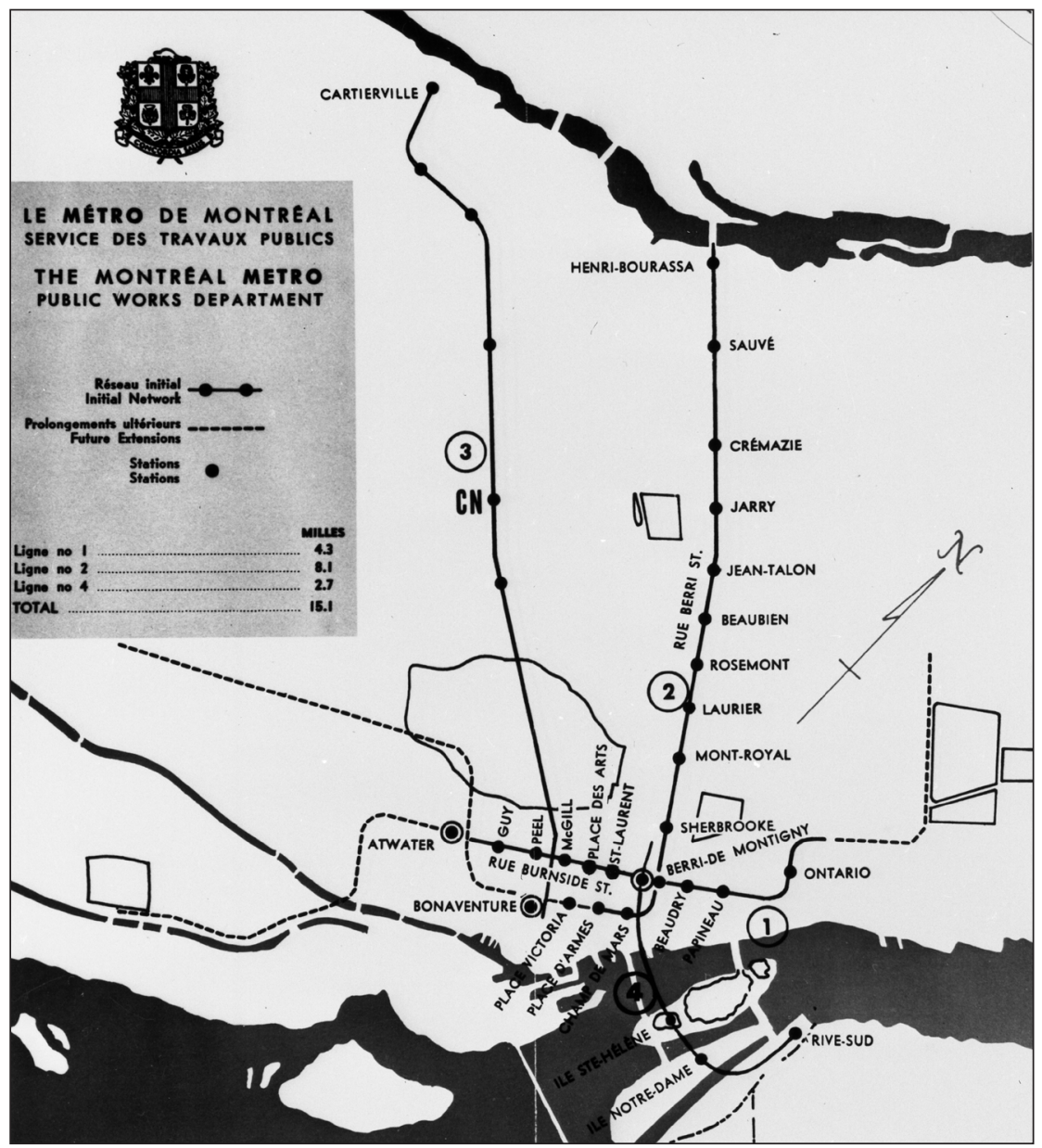

Source:AVM,VM94/M260-002.

desservant ces îles. Cette annonce cause la surprise tant au conseil municipal qu'au sein de la population, car parmi les rumeurs de prolongements, aucune ne faisait alors mention d'une telle liaison. Après avoir réfléchi à la possibilité de modifier le tracé de la ligne 2 pour lui faire traverser le fleuve plutôt que le quartier des affaires, les autorités montréalaises privilégient la création d'une ligne indépendante, reliée au reste du réseau à la station Berri-De Montigny, où se croisent les lignes 1 et $2^{62}$.

62. ASTM, S6, Série 7, Planification et coordination/Études des stations/Stations de la ligne 4 (2.3,20), mémo du comité d'étude du trafic, 24 avril 1963. Legault écrit que certains urbanistes proposèrent que 
Saulnier justifie cet ajout au réseau devant le conseil municipal par la nécessité d'un lien rapide et efficace entre l'île de Montréal et le site de l'Exposition afin de faciliter les déplacements des milliers de visiteurs attendus. En réponse aux critiques de certains conseillers dénonçant le " cadeau $^{63}$ " ainsi fait aux résidants de la Rive-Sud, il soutient que cette ligne s'avérera un bon investissement. Le président du Comité exécutif considère en effet qu'en vertu de l'achalandage prévu durant l'Exposition, cette ligne s'autofinancera sans problème et encouragera de plus les résidants de la Rive-Sud à venir magasiner régulièrement à Montréal.

Ainsi, le 11 juin 1963, Saulnier annonce devant le conseil municipal une série de modifications au réseau de métro alors en construction (figure $4)^{64}$. La branche nord-est de la ligne 3 devant se diriger vers Ahuntsic est définitivement éliminée, car sa mise en service nécessite la construction de voies ferrées additionnelles, afin de faciliter la cohabitation des rames de métro et des trains de passagers et de marchandises. La réalisation des autres segments de la ligne 3 est quant à elle reportée à une date ultérieure non déterminée. Le président du Comité exécutif justifie cette décision par le fait, d'une part, que les études en vue de l'adaptation des voies ferrées pour y faire rouler des voitures sur pneus sont toujours en cours, tout comme les pourparlers avec le CN. Il soutient, d'autre part, qu'il fut impossible de conclure une entente avec les municipalités voisines pour l'aménagement et le financement des stations. Saulnier souligne que ce manque de coopération, qu'il dénoncera à de nombreuses reprises par la suite, complexifie les négociations avec le $\mathrm{CN}^{65}$. Bien que la ligne 3 ne soit pas officiellement abandonnée, Drapeau et Saulnier ne manifesteront désormais que peu d'intérêt à son égard, réitérant périodiquement la nécessité d'une participation financière des municipalités de banlieue pour qu'elle puisse être réalisée.

Saulnier annonce également le 11 juin la construction d'une ligne 4 (actuelle ligne jaune) entre Montréal et Longueuil sur la rive sud du fleuve. Sa mise en service est prévue à temps pour l'inauguration de l'Exposition universelle. Alors qu'il dénonce le manque de collaboration des autorités

la station terminus à Montréal soit située plus à l'ouest. Les autorités, désireuses de développer un nouveau pôle d'activité dans l'est du centre-ville, optèrent cependant pour Berri-De Montigny, autre décision illustrant le fait que l'administration municipale accorda au métro un rôle de levier de croissance urbaine. G. Legault, La ville qu'on a bâtie..., op. cit., 55.

63. «Extension of Subway Justified», Montreal Star, 2 avril 1963.

64. Aucun des tronçons dont la construction a débuté ne se voit alors condamné.

65. Raymond Masse, «La ligne Berri prolongée à ses deux extrémités», La Presse; Jean-Marc Laliberté, "Le métro sera prolongé dans trois directions», Le Devoir, 12 juin 1963. 
des municipalités traversées par la ligne 3, le bras droit du maire souligne l'ouverture des autorités des municipalités de la Rive-Sud à contribuer d'une façon ou d'une autre à la réalisation de cette ligne ${ }^{66}$.

Saulnier confirme, par ailleurs, qu'à la suite d'une analyse concluant à la mauvaise qualité des sols sous la rue Craig, la ligne 2 empruntera l'axe de la rue Vitré, sensiblement à égale distance de la rue Saint-Jacques et du boulevard Dorchester. Il annonce également que cette ligne est prolongée à ses extrémités. Deux stations au nord, Sauvé et Henri-Bourassa, et deux autres à l'extrémité sud-ouest au centre-ville, Square-Victoria et Bonaventure, sont ainsi ajoutées, en conformité avec ce qui avait été évoqué depuis deux ans ${ }^{67}$. L'ajout des stations Sauvé et Henri-Bourassa, au coût de 6,1 millions de dollars, permet de préserver la desserte du secteur Ahuntsic malgré l'élimination de la branche nord-est de la ligne 3, et ce, à un coût inférieur, l'aménagement de cette branche ayant été évalué à 10 millions de dollars. Quant au prolongement au centre-ville, il assure la desserte des secteurs très fréquentés du square Victoria, près duquel on élève alors la Tour de la Bourse ${ }^{68}$, et des gares ferroviaires Centrale et Windsor, où transitent notamment des milliers de banlieusards. Le 6 août 1963, le conseil municipal approuve les crédits supplémentaires requis par ces modifications. Le coût total du réseau initial de métro est ainsi porté à 213,7 millions de dollars.

\section{UNE EXTENSION PROJETÉE VERS L'ÎLE JÉSUS}

Au moment de l'annonce du 11 juin 1963 par ailleurs, des pourparlers sont en cours entre des représentants de Montréal et des délégués de l'île Jésus, située au nord de l'île de Montréal. Une étude sur la faisabilité du prolongement de la ligne 3 de Cartierville jusqu'à la municipalité de Lavaldes-Rapides afin d'y construire une station jouxtant l'autoroute des

66. Les autorités longueuilloises confirment leur contribution financière de 3,3 millions de dollars en mars 1964. L'aménagement des abords de la station Longueuil est l'objet d'un travail de planification par les autorités municipales locales. Ces dernières voient dans cette station le fer de lance du développement résidentiel et commercial du secteur relativement désert où elle est construite. Le développement prévu se concrétise rapidement. L'édifice résidentiel Port-de-Mer et le complexe de bureaux Montval, par exemple, sont inaugurés en 1968.

67. Lors du dévoilement du projet en octobre 1961, Saulnier et Drapeau avaient en effet mentionné que la desserte du square Victoria et des gares ferroviaires Centrale et Windsor était prévue rapidement, ces deux stations étant même indiquées sur la carte du réseau (figure 3). Le prolongement de la ligne 2 avait également été évoqué par Saulnier au printemps 1962 au moment où l'administration avait découvert que le montant des soumissions en vue de la construction des premiers tronçons du réseau était beaucoup moins élevé que prévu, résultat d'une concurrence féroce entre les entrepreneurs selon Gignac. Benoît Gignac, Le maire qui rêvait sa ville. Jean Drapeau (Montréal, Éditions La Presse, 2009), 126.

68. Inaugurée en juillet 1964. 
Laurentides avait en effet soulevé l'intérêt d'élus et d'hommes d'affaires locaux au début de l'année 1963 et mené au lancement de discussions ${ }^{69}$. À la mi-juin, après l'annonce des modifications au réseau de métro, des représentants de Duvernay se joignent aux discussions. Profitant du report de la ligne 3 et soulignant, comme leurs collègues de Laval-des-Rapides, la nécessité de faciliter les déplacements entre Montréal et les municipalités de l'île Jésus, qui sont alors en croissance démographique rapide, ils proposent que la ligne 2 soit prolongée de la station Henri-Bourassa jusqu'au territoire de leur municipalité. Ils vantent pour ce faire la position centrale de Duvernay sur l'île Jésus ainsi que la présence de terrains susceptibles d'accueillir une station et un vaste parc de stationnement ${ }^{70}$. Ces représentants voient bientôt les autorités de Pont-Viau rejoindre les pourparlers.

En octobre 1963, un projet de prolongement de la ligne 2 d'un peu plus d'un kilomètre, entre la station Henri-Bourassa et un terrain vacant près de l'hôtel de ville de Pont-Viau, rallie les divers interlocuteurs. Ce terrain jouit d'une position avantageuse, car il se situe entre Laval-des-Rapides et Duvernay, au cœur d'un secteur fort populeux de l'île Jésus. Les autorités de l'île engagées dans les pourparlers perçoivent cette liaison rapide vers le centre-ville de Montréal comme un excellent gage de la poursuite de la croissance de leurs municipalités. Leurs vis-à-vis montréalais voient également d'un très bon œil la possibilité d'établir le terminus de la ligne 2 hors du territoire de Montréal. L’aménagement d'un parc de stationnement pour les automobilistes désireux d'effectuer un transfert modal, afin d'éviter la congestion routière sur l'île de Montréal, apparaît en effet plus judicieux à Pont-Viau qu'aux abords de la station Henri-Bourassa dans le quartier Ahuntsic, où la marge de manœuvre est considérablement plus limitée. Drapeau et Saulnier manifestent ainsi un vif intérêt pour ce court prolongement.

Le financement de ce projet par les municipalités de l'île Jésus constituant une pierre d'achoppement des discussions, le maire de Pont-Viau, Roger Provost, propose en janvier 1964 le partage de la facture entre Montréal et sa municipalité, peuplée alors d'un peu moins de 20000 habitants ${ }^{71}$. Provost estime que Pont-Viau est en mesure de débourser deux millions de dollars considérant le développement qu'entraînera la

69. Jean-Louis Arcand, «Un nouveau projet: deux importants prolongements», La Presse, 9 mars 1963.

70. Jean-Louis Arcand, «Duvernay veut une station de métro», La Presse, 19 juin 1963.

71. Jean-Charles Fortin et al., Histoire de Laval (Québec, PUL, 2008), 189. 
présence du métro ${ }^{72}$. La mise sur pied de la Commission d'étude sur les problèmes intermunicipaux de l'île Jésus, le 6 février 1964, cause cependant l'arrêt des négociations, l'administration montréalaise ne souhaitant pas conclure une entente qui pourrait être rendue caduque, ou du moins remise en question, par la fusion des municipalités de l'île. Ce projet de fusion est en effet étudié par la Commission, et il est mis en ouvre après le dépôt du rapport de cette dernière en janvier 1965.

La création de la Ville de Laval le 6 août 1965 fragilise le projet de prolongement du métro vers l'île Jésus. Le financement d'une courte extension d'une seule station ne desservant qu'une petite partie de l'île apparaît en effet difficilement justifiable sur le plan politique pour les nouvelles autorités lavalloises, alors que ce projet est le scénario privilégié par l'administration montréalaise. Plusieurs citoyens, conseillers municipaux et éditorialistes, pour leur part, remettent en question à Montréal le bienfondé de ce prolongement, alors que la desserte de quartiers populeux comme Saint-Henri ou Notre-Dame-de-Grâce, constituant des bassins importants d'usagers du transport collectif et notamment d'usagers de statuts socio-économiques modestes, ne semble pas à l'ordre du jour.

En février 1966, Saulnier déclare qu'un prolongement à Laval ne pourra pas avoir lieu tant que le réseau de métro ne sera pas davantage développé sur l'île de Montréal. Il ajoute du même souffle que la ligne 4 ne doit son existence qu'à la tenue d'un événement exceptionnel, l’Exposition universelle $^{73}$. À l'approche du scrutin municipal, l'administration tient manifestement à démontrer qu'elle prend acte des demandes de la population montréalaise. La déclaration de Saulnier consacre ainsi le report du projet de prolongement à Laval ${ }^{74}$.

\section{CONCLUSION}

Après quatre ans de travaux et un peu plus d'une semaine avant les élections municipales, les Montréalais montent à bord du métro le 14 octobre $1966^{75}$. Ce réseau planifié par les autorités municipales (figure 5) témoigne non seulement d'une volonté de soulager la congestion, mais également d'autres visées, qui révèlent divers rapports au territoire. Tout en tenant

\footnotetext{
72. Jean-Louis Arcand, "Seul Pont-Viau collaborera au financement du métro», La Presse, 30 janvier 1964.

73. T. D., "Le prolongement du métro via le tunnel mont Royal devient un projet complexe», $L a$ Presse, 24 février 1966.

74. Il ne se concrétisera qu'au début des années 2000.

75. La ligne 4 ainsi que les stations Frontenac, Beaudry, Square-Victoria et Bonaventure ne sont alors pas encore prêtes; elles accueilleront leurs premiers passagers au cours des mois suivants.
} 
Figure 5

Réseau initial de métro, 1967

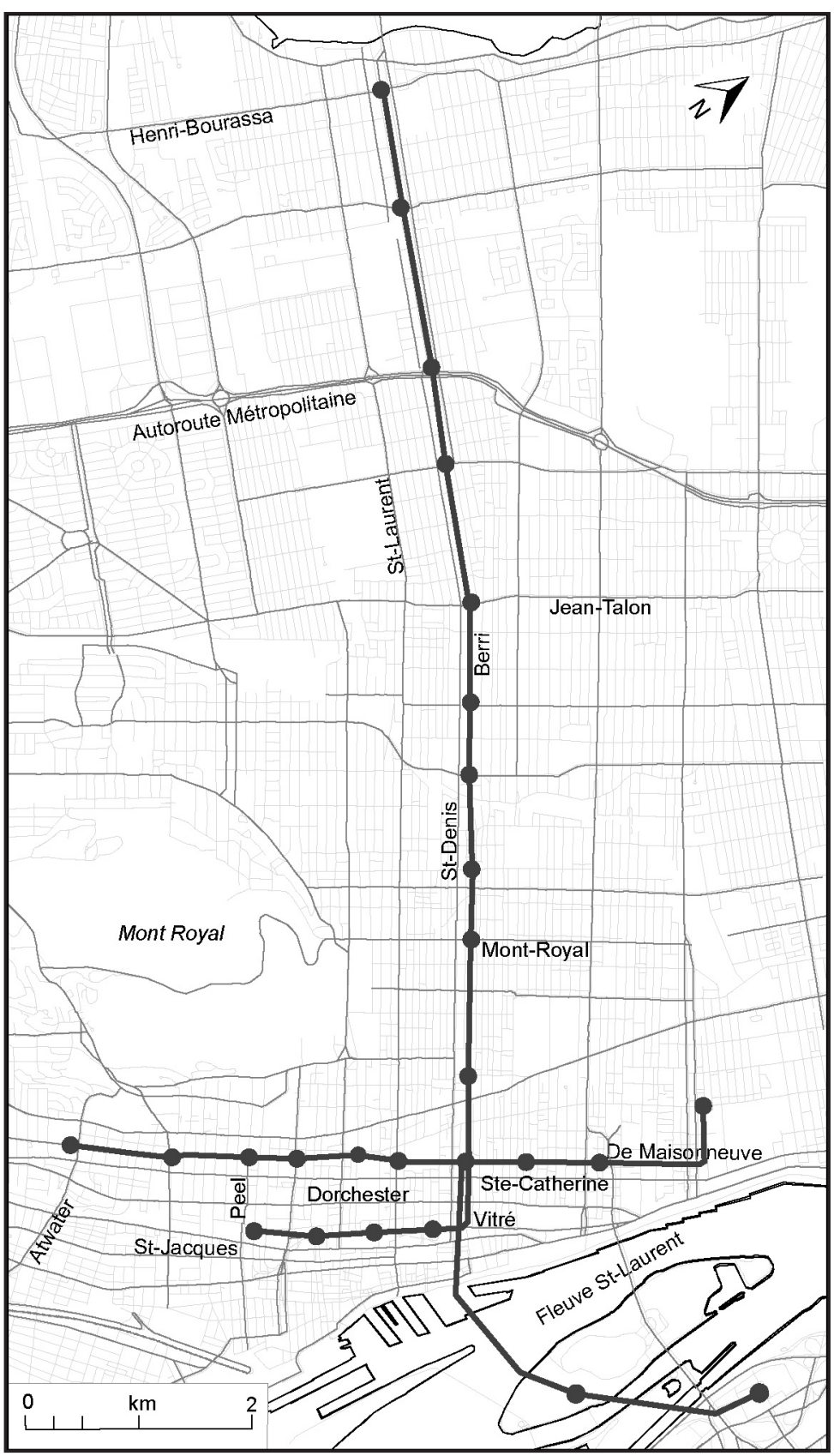

Source:ASTM, S6/10.1.5,3. 
compte de la nécessité d'une desserte efficace des bassins d'usagers du transport collectif, gage de rentabilité, et d'impératifs techniques incontournables comme la nature des sols, l'administration Drapeau-Saulnier endossa la position défendue par les professionnels du Service d'Urbanisme. Le métro fut ainsi appelé à servir de vecteur d' "urbanogenèse ${ }^{76}$ " dans certaines zones dévalorisées et à appuyer l'émergence de nouveaux pôles d'activité au centre-ville. La protection des artères majeures de la ville apparaît de même comme un facteur important dans les alignements choisis au moment où en Amérique du Nord les rues marchandes commencent à perdre de leur superbe face aux nouveaux temples de la consommation et où la croissance économique de Montréal connaît des ratés. On décida de soustraire les rues Sainte-Catherine, Saint-Denis et Saint-Jacques aux inconvénients d'un long chantier de construction pour ne pas affecter leur vitalité et, par ricochet, celle de la ville en entier ${ }^{77}$.

Le territoire d'action des autorités en charge du projet, leur important souci du coût des travaux, de même que l'application d'une stratégie de développement par étapes expliquent certes pourquoi le réseau mis en service en 1966 fut essentiellement situé sur le territoire montréalais. Néanmoins, ce réseau se veut également le reflet des tensions politiques à l'échelle métropolitaine. La volonté de l'administration montréalaise, peu portée sur la concertation régionale, d'obtenir le droit d'opérer librement sur le territoire des municipalités d'Outremont, de Mont-Royal et de Saint-Laurent suscita l'opposition de ces dernières. Cette opposition, tout comme le choix du système de roulement sur pneumatiques, contribuèrent à l'annulation du projet de ligne 3 . Au final, la réalisation et la planification de tronçons à l'extérieur des limites municipales s'expliquent moins par le désir des autorités montréalaises de voir le métro participer à la structuration du territoire métropolitain, en termes de connectivité et de développement, que par leur volonté de servir d'abord et avant tout les intérêts de Montréal. Elles furent en effet soucieuses de desservir adéquatement les quartiers du nord de la ville et le site de l'Exposition universelle et intéressées à transférer en banlieue les charges de circulation et de stationnement associées aux stations situées en bout de ligne.

76. Jacques Lévy, «Modèle de mobilité, modèle d'urbanité», dans François Ascher et al., dir., Les sens du mouvement (Paris, Belin, 2004), 157.

77. La vitalité de la rue Saint-Jacques sera néanmoins affectée par le déplacement des pôles d'activité au centre-ville au cours des années suivantes, alors que les rues Sainte-Catherine et Saint-Denis souffriront de la multiplication des centres commerciaux. 
Bien que boudé par plusieurs automobilistes pour qui revenir ou migrer vers le transport collectif était hors de question, le métro fut investi par des milliers de Montréalais dès son inauguration, entraînant une hausse de l'achalandage de la CTM. Sa présence et son utilisation eurent un impact - demeurant à approfondir - sur leur pratique de l'espace urbain et leurs représentations de Montréal. Élevée au rang de symbole de l'affirmation de la ville comme métropole d'envergure internationale par l'administration Drapeau, l'arrivée du métro, comme l' «Expo», permirent d'occulter la morosité grandissante de l'économie montréalaise. La situation économique allait néanmoins être pointée du doigt par Lucien L'Allier en 1969 pour expliquer le retour à la baisse du nombre d'usagers de la $\mathrm{CTM}^{78}$. 